\title{
Energization of Charged Particles to High Energies by an Induced Substorm Electric Field within the Magnetotail
}

\author{
W.J. Heikkila, ${ }^{*}$ R.J. Pellinen, ${ }^{* *}$ and P.O. Welling ${ }^{* *}$ \\ *The University of Texas at Dallas, Richardson, Texas, U.S.A. \\ **Finnish Meteorological Institute, Helsinki, Finland
}

(Received October 17, 1977)

Numerous observations have been reported of bursts of electrons and protons with $\mathrm{MeV}$ energies in the magnetotail. The purpose of this paper is to propose a mechanism for their acceleration.

Since super-Alfvenic drifts are unlikely in the magnetotail, an upper limit to the electric field can be obtained, probably less than $10 \mathrm{mV} / \mathrm{m}$. Any mechanism for energizing charged particles involving steady state electric fields, such as driftbetatron acceleration, is thus subject to a stringent condition, namely the total available potential difference. Gyration in an induced electric field, with a finite electromotive force, does not have this limitation. It is impossible for polarization of charge in a plasma to remove the electromotive force of an induced electric field; in removing the parallel component the polarization must enhance the transverse

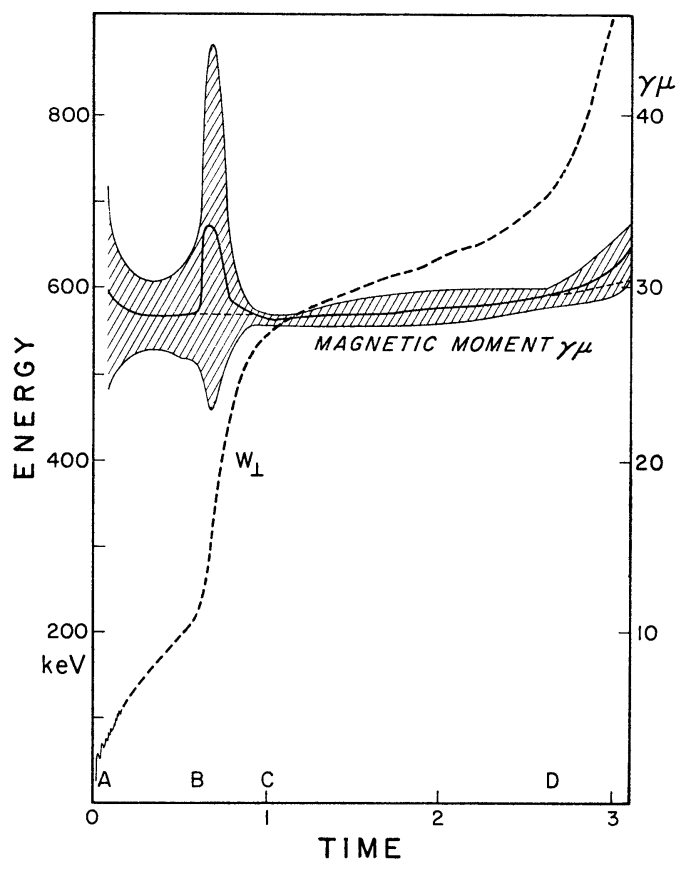

Fig. 1 
component. We know from observations that the tail magnetic field exhibits large variations in time, which necessarily must involve induction fields. Hence, it is appropriate to start from an assumed time-dependent perturbation near the neutral sheet, compute the corresponding fields, and then make orbit calculations to see if some particles might gain sufficient energy.

The assumed perturbation model is as shown in Heik kila and Pellinen (1977); the perturbation is assumed to grow linearly in time and space. One example of our calculations is shown in Fig. 1. This electron orbit is near an O-type neutral line, due to an increased cross tail current density. The electron is first accelerated along the O-line by the electric field; it thus gains about $50 \mathrm{keV}$ through simple linear acceleration, as shown at point $\mathrm{A}$. By means of this linear acceleration it is possible for the particle to start out with a large magnetic moment when it becomes trapped in the magnetic field. Thereafter, it is betatron-accelerated up to about $1 \mathrm{MeV}$, keeping the relativistic first invariant almost constant. Particles starting near the X-line will also experience a similar two-step acceleration process. Particles starting at some point away from the neutral sheet will also be accelerated along the magnetic field due to $E_{11}$, leading to a cyclic variation of electron and proton precipitation as is observed in substorms.

This research was supported by the Swedish Natural Science Research Council, and the U.S. National Science Foundation.

\section{REFERENCE}

Heiknila, W.J. and R.J. Pellinen, Localized induced electric field within the magnetotail, $J$. Geophys. Res., 82, 1610-1614, 1977. 